\title{
Baseline cardiovascular comorbidities, and the influence on cancer treatment decision-making in women with breast cancer
}

\author{
Shridevi Subramaniam ${ }^{1,2}$, Yek-Ching Kong ${ }^{1,3}$, Hafizah Zaharah4 ${ }^{4}$ Cuno S.P.M. Uiterwaal5 ${ }^{5}$, Andrea Richard², Nur Aishah Taib ${ }^{6}$, Azura Deniel7, \\ Kok-Han Chee ${ }^{8}$, Ros Suzanna Bustamam7, Mee-Hoong See ${ }^{6}$, Alan Fong ${ }^{9}$, Cheng-Har Yip ${ }^{10}$ and Nirmala Bhoo-Pathy ${ }^{1,3}$
}

${ }^{1}$ Department of Social and Preventive Medicine, Faculty of Medicine, Universiti Malaya, 50603 Lembah Pantai, Kuala Lumpur, Malaysia

${ }^{2}$ Centre of Clinical Epidemiology, Institute for Clinical Research, National Institutes of Health, Setia Alam, 40170 Shah Alam, Malaysia

${ }^{3}$ Centre for Epidemiology and Evidence-Based Practice, Faculty of Medicine, Universiti Malaya, 50603 Lembah Pantai, Kuala Lumpur, Malaysia

${ }^{4}$ Department of Radiotherapy \& Oncology, National Cancer Institute, 62250 Putrajaya, Malaysia

${ }^{5}$ Julius Center for Health Sciences and Primary Care, University Medical Center Utrecht, 3508 GA Utrecht, The Netherlands

${ }^{6}$ Department of Surgery, Faculty of Medicine, Universiti Malaya, 50603 Lembah Pantai, Kuala Lumpur, Malaysia

${ }^{7}$ Department of Radiotherapy and Oncology, Hospital Kuala Lumpur, 50586 Kuala Lumpur, Malaysia

${ }^{8}$ Cardiology Unit, Department of Medicine, Faculty of Medicine, Universiti Malaya, 50603 Lembah Pantai, Kuala Lumpur, Malaysia

${ }^{9}$ Sarawak Heart Centre, 94300 Kota Samarahan, Sarawak, Malaysia

${ }^{10}$ Subang Jaya Medical Centre, 47500 Subang Jaya, Selangor, Malaysia

\section{Abstract}

Purpose: To measure the baseline prevalence of cardiovascular disease (CVD), its modifiable and non-modifiable risk factors in breast cancer patients, and determine their association with adjuvant treatment decision-making.

Method: From 2016 to 2017, 2,127 women newly-diagnosed with breast cancer were prospectively recruited. Participants' cardiovascular biomarkers were measured prior to adjuvant treatment decision-making. Clinical data and medical histories were obtained from hospital records. Adjuvant treatment decisions were collated 6-8 months after recruitment. A priori risk of cardiotoxicity was predicted using the Cardiotoxicity Risk Score.

Results: Mean age was 54 years. Eighty-five patients had pre-existing cardiac diseases and 30 had prior stroke. Baseline prevalence of hypertension was $47.8 \%$. Close to $20 \%$ had diabetes mellitus, or were obese. Dyslipidaemia was present in $65.3 \%$. The proportion of women presenting with $\geq 2$ modifiable CVD risk factors at initial cancer diagnosis was substantial, irrespective of age. Significant ethnic variations were observed. Multivariable analyses showed that pre-existing CVD was consistently associated with lower administration of adjuvant breast cancer therapies (odds ratio for chemotherapy: 0.32 , 95\% confidence interval: 0.17-0.58). However, presence of multiple risk factors of CVD did not appear to influence adjuvant treatment decision-making. In this study, $63.6 \%$ of patients were predicted to have high risks of developing cardiotoxicities attributed to a high baseline burden of CVD risk factors and anthracycline administration.

Conclusion: While recent guidelines recommend routine assessment of cardiovascular comorbidities in cancer patients prior to initiation of anticancer therapies, this study highlights the prevailing gap in knowledge on how such data may be used to optimise cancer treatment decision-making.

Keywords: breast cancer, cardiovascular risk factors, cardiotoxicity

Correspondence to: Nirmala Bhoo Pathy. Email: ovenjjay@gmail.com

ecancer 2021, 15:1293

https://doi.org/10.3332/ecancer.2021.1293

Published: $21 / 09 / 2021$

Received: 25/02/2021

Publication costs for this article were supported by ecancer (UK Charity number 1176307).

Copyright: (c) the authors; licensee ecancermedicalscience. This is an Open Access article distributed under the terms of the Creative Commons Attribution License (http:// creativecommons.org/licenses/by/4.0), which permits unrestricted use, distribution, and reproduction in any medium, provided the original work is properly cited. 


\section{Introduction}

Cardiovascular disease (CVD) is emerging to rival cancer recurrence as a leading cause of death in women with early breast cancer [1].The increased risk of morbidity and mortality from CVD in women with breast cancer may be attributed to a combination of the direct cardiotoxic effects (e.g. anthracycline-based chemotherapy, trastuzumab, radiotherapy) and the indirect effects (e.g. weight gain, loss of cardiorespiratory fitness) of cancer therapy, coupled with clustering of conventional risk factors of CVD including hypertension, diabetes mellitus, dyslipidaemia, obesity and smoking [2-8].

Until very recently, guidelines for cardiovascular risk assessment in adults with cancer had been scarce [9-10]. Particularly, routine screening for conventional risk factors of CVD at initial cancer diagnosis had been lacking in oncology practices worldwide [9] despite its potential to facilitate cardiovascular profiling, adjuvant treatment decision-making and identification of high-risk patients requiring early cardiology referral and close monitoring [11]. In recognition of the increasing need for guidance, the American Society of Clinical Oncology had in 2017 put up a specific guideline for cardiovascular care of cancer patients, which was endorsed by the American College of Cardiology and the American Heart Association. This guideline recommends physicians to perform comprehensive cardiovascular evaluation in adult patients with cancer including screening for modifiable risk factors of CVD before initiation of potentially cardiotoxic therapies [12-13].

Through an inception cohort study, we measured the prevalence of CVD, its modifiable (hypertension, diabetes, obesity, dyslipidaemia, smoking) and non-modifiable (family history) risk factors, prior to administration of anticancer therapies in women newly diagnosed with breast cancer in a multiethnic setting. Importantly, amidst the lack of evidence-based standards, we assessed whether the baseline presence of CVD or presence of multiple risk factors of CVD was associated with adjuvant treatment administration, to gain an insight on whether these factors currently influenced cancer treatment decision-making in routine clinical practice. A priori risk of cardiotoxicity following adjuvant therapy was also predicted.

\section{Patients and methods}

\section{Study population}

The study population comprised women who were newly diagnosed with stage I up to stage IV breast cancer between January 2016 and December 2017. Four different hospitals were selected across Klang Valley, an urban conglomeration in Malaysia, to allow recruitment of a demographically diverse sample of patients; National Cancer Institute (national oncology referral center), Kuala Lumpur Hospital (public general hospital), University Malaya Medical Centre (public university hospital) and Subang Jaya Medical Centre (private medical centre). In total, 2,127 females with a first time histological-diagnosis of breast cancer were consecutively recruited within 6-8 weeks of diagnosis, prior to initiation of cancer therapy. Patients with recurrent breast cancer were excluded.

\section{Ethical approval}

Ethics approval was obtained from the relevant institutional committees. Written informed consent was obtained from all participants. Ethical principles according to Helsinki's declaration were observed throughout the conduct of the study.

\section{Data collection and study variables}

Participants were recruited during their hospital visits. Data on ethnicity (Malay, Chinese, Indian or other race), age at diagnosis, highestattained education (primary, secondary or tertiary) and medical history (prior diagnosis with hypertension, diabetes mellitus, dyslipidaemia, eCVDs (myocardial infarction, angina pectoris, unstable angina, heart failure and stroke), other comorbidities and medications) were collected via face-to-face interviews. Data on smoking status and family history of premature heart disease (history of coronary heart disease before the age of 60 years in first-degree relatives) were also gathered. All medical and drug histories were verified with medical records. 
During the on-site screenings, height and body weight were measured. Body mass index (BMI) was calculated as weight divided by height squared $\left(\mathrm{kg} / \mathrm{m}^{2}\right)$. Obesity was recorded if the calculated BMI was $30 \mathrm{~kg} / \mathrm{m}^{2}$ and above. Systolic and diastolic blood pressure readings were taken using automated sphygmomanometers. Hypertension was recorded based on self-report or if patients were on hypertensive medication and/or had a repeatedly elevated BP reading; systolic blood pressure $>140 \mathrm{mmHg}$ or diastolic blood pressure $>90 \mathrm{mmHg}$ (measured at least twice).

Non-fasting blood samples were drawn to measure lipid and glucose levels. Dyslipidaemia was recorded based on self-report or if patients were on cholesterol lowering medication and/or had a serum total cholesterol level $\geq 5.2 \mathrm{mmol} / \mathrm{L}$ and/or serum high density lipoprotein levels $(\mathrm{HDL})<1.3 \mathrm{mmol} / \mathrm{L}$ [14]. Diabetes status was recorded based on self-report or if patients were on diabetes medication and/or had a non-fasting blood glucose concentration of $\geq 11.0 \mathrm{mmol} / \mathrm{L}$.

Patients who were newly detected with abnormalities in their blood pressure or serum glucose/lipid levels were provided with referral letters for further management.

Data on tumour characteristics were obtained from hospital records (date of diagnosis, histology, tumour size at presentation, number of positive axillary lymph nodes, tumour grade (grade I, grade II, grade III), distant metastasis at initial diagnosis (yes or no), TNM stage (I, II, III, IV), progesterone and oestrogen receptor status (positive, negative) and human epidermal growth factor receptor 2 status (positive, negative)).

\section{Follow-up for adjuvant treatment administration, and prediction of cardiotoxicity}

Patients' baseline data on risk factors of CVD were accessible through the medical records to all treating physicians prior to adjuvant treatment decision-making. Data on administration of type of surgery, radiotherapy, chemotherapy, chemotherapy regimen, endocrine therapy, type of endocrine therapy, targeted therapy and type of targeted therapy were retrieved from the hospital records within 6-8 months after recruitment.

Individual risk of cardiotoxicity was subsequently calculated using the Cardiotoxicity Risk Score, a risk-stratification tool introduced by the Mayo Clinic [11] that allows physicians to assign cancer patients into very low, low, intermediate, high and very high cardiotoxicity risk groups based on a set of patient-and cancer treatment-related risk factors. Under this scoring system, one point was assigned for a medical history of hypertension, diabetes, cardiomyopathy or heart failure, having a history of coronary artery disease, age below 15 or above 65 years, being female, prior or concurrent anthracycline-based chemotherapy and prior or concurrent chest radiation. Up to four points were assigned if patients received cardiotoxic drugs such as cyclophosphamide, anthracycline or trastuzumab. A total score of less than two was considered to indicate a low risk of cardiotoxicity, while a score of three to four represented an intermediate risk, a score of five to six was deemed high risk and a score of more than six denoted a very high risk [11].

\section{Data analysis}

Categorical variables were summarised using frequency and percentages. Continuous variables were expressed in mean \pm standard deviation. Overall prevalence of cardiovascular comorbidities with $95 \%$ confidence intervals (Cls) was estimated for participants, followed by stratification by age and ethnicity.

Among patients with non-metastatic disease, multivariable logistic regression analyses were used to determine the association between preexisting CVD, family history of premature heart disease and clustering of risk factors of CVD (presence of $\geq 2$ modifiable risk factors) with administration of individual modalities of adjuvant treatment. Models were adjusted for age (years), ethnicity (Chinese, Malay, Indian, others), type of hospital (public, private, university), tumour size (pT1, pT2, pT3, pT4), lymph node status (pN0, pN1, pN2, pN3), oestrogen/progesterone receptor status (positive, negative), human epidermal growth factor receptor 2 status (positive, negative), tumour grade (I, II, III), adjuvant chemotherapy administration (no, yes), adjuvant radiotherapy administration (no, yes), adjuvant endocrine therapy administration (no, yes) and trastuzumab administration (no, yes).

Complete case analyses were performed, and multiple imputation was employed as a form of sensitivity analysis. 


\section{Results}

In this large cohort of multiethnic Asian women (Chinese: $47.9 \%$, Malays: $37.4 \%$, Indians: $12.7 \%$ ) with breast cancer, the mean age at diagnosis was 54 years (11.6). Mean tumour size at presentation was $3.6 \mathrm{~cm}$ (2.9). Overall, $22.4 \%$ presented with stage I disease, while $38.9 \%$, $27.9 \%$ and $10.8 \%$ presented with stage II, stage III and stage IV breast cancers, respectively. A vast majority (70\%) had oestrogen receptor positive tumours. Among 1,876 patients with non-metastatic breast cancer, $63.1 \%$ received chemotherapy (53.0\%; anthracycline- and taxane-based regimens, 41.4\%; anthracyclines only, 5.2\%; taxanes alone), $63.1 \%$ received adjuvant radiotherapy, while approximately $20 \%$ of patients (126/618) with human epidermal growth factor receptor 2 (HER2) positive tumours received trastuzumab (not shown). Missing values ranged between $0.2 \%$ (diabetes mellitus) and $12.1 \%$ (tumour grade).

Eighty-five patients had preexisting cardiac diseases $(4.0 \%, 95 \% \mathrm{Cl}: 3.2 \%-4.8 \%)$. Of these, a majority comprised coronary heart disease $(n=$ 75), with 31 patients having suffered a previous myocardial infarction (Table 1). Thirty patients had a prior history of stroke. Approximately $11 \%$ of patients reported a family history of premature coronary heart disease. Very few patients were classified as having a cardiovascular risk factor based on self-report alone (hypertension: 15 patients, dyslipidaemia: 17). The baseline prevalence of hypertension in this cohort of women with newly diagnosed breast cancer was $47.8 \%$ (95\% Cl: $45.7 \%-49.9 \%$ ) (Table 1). Close to $20 \%$ of patients had diabetes mellitus at initial breast cancer diagnoses (18.8\%, 95\% Cl: 17.1\%-20.5\%). Prevalence of obesity at baseline was 18.0\% (16.3\%-19.6\%). A majority (65.3\%) of patients presented with dyslipidaemia; $44.7 \%$ (95\% Cl: $42.3 \%-47.1 \%)$ had elevated total cholesterol, 38.1\% (95\% Cl: $35.7 \%-40.4 \%$ ) had low HDL levels (not mutually exclusive) (not shown). Only a minority reported smoking (1.7\%, 95\% Cl: 1.1\%-2.2\%). In this relatively young cohort of multiethnic women with breast cancer, the clustering of risk factors of CVD was substantial where $26.3 \%$ of patients presented with two modifiable risk factors at baseline, while another $20.7 \%$ already had three risk factors at initial breast cancer diagnosis. Strikingly, the clustering of CVD risk factors was not negligible even among the very young breast cancer patients. All the cardiovascular profiles were significantly associated with age ( $p$ value $<0.05$ ) except smoking and family history of early-onset coronary heart disease (Table 1). Significant ethnic variations were observed, whereby Indian women with breast cancer were more likely to have preexisting CVD, diabetes, hypertension, dyslipidaemia and family history of early-onset coronary heart disease compared to their Chinese and Malay counterparts ( $p$ value $<0.05$ ) (Table 2). Clustering of modifiable risk factors for CVD remained highest in Indian patients with breast cancer, with $63.6 \%$ presenting with two or more risk factors, compared to the Malays (54.4\%) and the Chinese (36.5\%).

In the multivariable analyses examining the association between cardiovascular profile and adjuvant treatment administration, presence of pre-existing CVD was significantly associated with lower odds of receiving chemotherapy (adjusted odds ratio (OR): 0.32, 95\% Cl: 0.170.58). None of the patients with pre-existing CVD received trastuzumab. Radiotherapy or endocrine therapy administrations were also less likely in women with pre-existing CVD, albeit not reaching statistical significance (Table 3). Interestingly, the presence of multiple modifiable risk factors of CVD at initial diagnosis was not associated with administration of most adjuvant therapies except endocrine therapy (Table 3 ).

Using the Cardiotoxicity Risk Score, approximately one fifth of breast cancer patients were predicted to have low risk of cardiotoxicity (19.9\%), while a majority appeared to have high (33.7\%) or very high risks (29.9\%) of developing cardiotoxicities (Table 4). Patients aged <40 years, those of Malay and Indian ethnicities, as well as women with more advanced cancer stages, appeared to be more vulnerable. Multivariable analysis further revealed that younger age and advanced cancer stages remained independently associated with a higher predicted risk of cardiotoxicity.

Results from sensitivity analyses using multiple imputation did not change the study inferences.

\section{Discussion}

Findings from this multi-ethnic setting revealed a high baseline burden of modifiable risk factors of CVD among women presenting with breast cancer, including in those who were very young. While pre-existing CVD appeared to be consistently associated with lower administration of adjuvant breast cancer therapies, presence of multiple risk factors of CVD at initial cancer diagnosis did not seem to influence adjuvant treatment decision-making. This, however, must be considered in light of our findings that a high burden of cardiovascular comorbidities at baseline coupled with administration of anthracyclines puts women with breast cancer at high a priori risks of cardiotoxicity. 


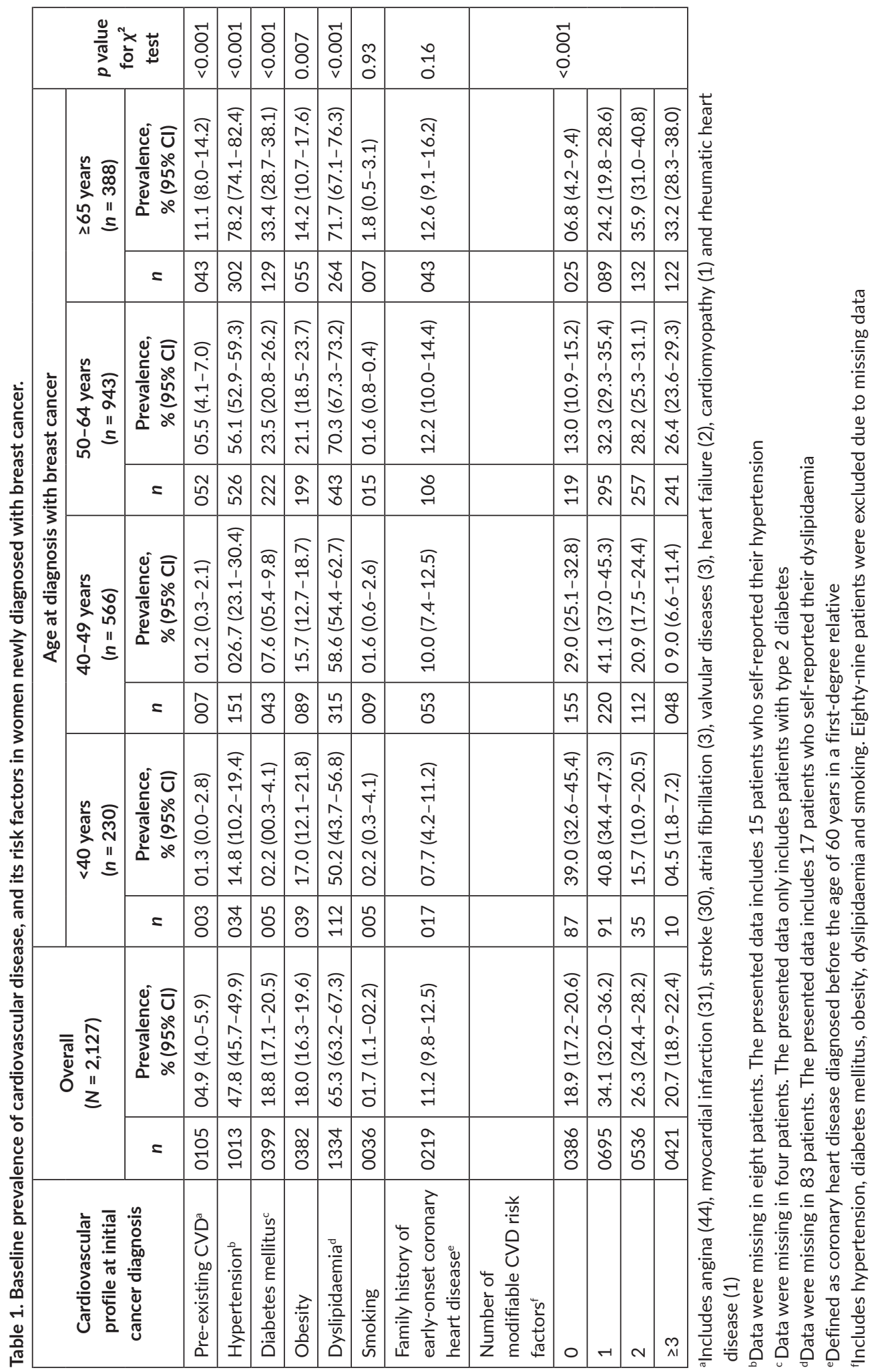


Table 2. Baseline prevalence of CVD, and its risk factors by ethnicity in women newly diagnosed with breast cancer.

\begin{tabular}{|c|c|c|c|c|c|c|c|}
\hline \multirow{3}{*}{$\begin{array}{l}\text { Cardiovascular profile at } \\
\text { initial cancer diagnosis }\end{array}$} & \multicolumn{6}{|c|}{ Ethnicity } & \multirow{3}{*}{$\begin{array}{c}p \text { value for } \chi^{2} \\
\text { test }\end{array}$} \\
\hline & \multicolumn{2}{|r|}{$\begin{array}{c}\text { Malay } \\
(n=795)\end{array}$} & \multicolumn{2}{|c|}{$\begin{array}{l}\text { Chinese } \\
(n=1,019)\end{array}$} & \multicolumn{2}{|r|}{$\begin{array}{c}\text { Indian } \\
(n=270)\end{array}$} & \\
\hline & $n$ & $\begin{array}{l}\text { Prevalence } \\
(95 \% \mathrm{Cl})\end{array}$ & $n$ & $\begin{array}{l}\text { Prevalence } \\
(95 \% \mathrm{CI})\end{array}$ & $n$ & $\begin{array}{l}\text { Prevalence } \\
(95 \% \mathrm{Cl})\end{array}$ & \\
\hline Preexisting $\mathrm{CVD}^{\mathrm{a}}$ & 46 & $05.8(4.2-7.4)$ & 35 & $03.4(02.3-4.6)$ & 22 & $08.1(04.9-11.4)$ & 0.002 \\
\hline Hypertension $^{b}$ & 424 & $53.3(49.9-56.8)$ & 409 & 40.5 (37.4-43.5) & 159 & $58.9(53.0-64.8)$ & $<0.001$ \\
\hline Diabetes mellitus $^{c}$ & 188 & $23.7(20.7-26.6)$ & 96 & $009.4(7.7-11.2)$ & 108 & $40.0(34.2-45.8)$ & $<0.001$ \\
\hline Obesity & 228 & $28.7(25.5-31.8)$ & 82 & $008.0(6.4-9.7)$ & 66 & $24.4(19.3-29.6)$ & $<0.001$ \\
\hline Dyslipidaemiad $^{d}$ & 517 & $65.7(62.4-69.0)$ & 599 & $63.1(60.0-66.2)$ & 194 & 72.9 (67.6-78.3) & 0.02 \\
\hline Smoking & 7 & $00.9(0.2-1.5)$ & 25 & $002.5(1.5-3.4)$ & 2 & $0.7(0.0-1.8)$ & 0.02 \\
\hline $\begin{array}{l}\text { Family history of early-onset } \\
\text { coronary heart disease }\end{array}$ & 102 & $14.1(11.6-16.7)$ & 71 & $007.5(05.8-9.2)$ & 41 & $16.1(11.6-20.7)$ & $<0.001$ \\
\hline \multicolumn{8}{|l|}{$\begin{array}{l}\text { Number of modifiable CVD } \\
\text { risk factors }^{f}\end{array}$} \\
\hline 0 & 133 & $16.9(14.3-19.5)$ & 217 & $23.0(20.3-25.7)$ & 27 & $10.2(6.5-13.8)$ & \multirow{4}{*}{$<0.001$} \\
\hline 1 & 226 & $28.7(25.6-31.9)$ & 382 & $40.5(37.4-43.6)$ & 70 & $26.3(21.0-31.6)$ & \\
\hline 2 & 210 & $26.7(23.6-29.8)$ & 243 & $25.8(23.0-28.6)$ & 76 & $28.6(23.1-34.0)$ & \\
\hline$\geq 3$ & 218 & $27.7(24.6-30.8)$ & 101 & $10.7(8.7-12.7)$ & 93 & $35.0(29.2-40.7)$ & \\
\hline
\end{tabular}

ancludes angina, myocardial infarction, stroke, atrial fibrillation, valvular diseases, heart failure, cardiomyopathy and rheumatic heart disease

${ }^{b}$ Data missing in eight patients. The presented data includes 15 patients who self-reported their hypertension

cData missing in four patients

${ }^{\mathrm{d}}$ Data missing in 82 patients. The presented data includes 17 patients who self-reported their dyslipidaemia

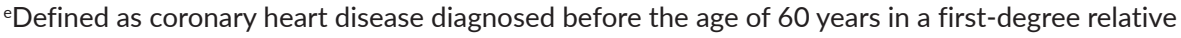

fIncludes hypertension, diabetes mellitus, obesity, dyslipidaemia and smoking. Eighty-eight patients were excluded due to missing data for any of the risk factors

The baseline prevalence of hypertension, diabetes and dyslipidaemia among the multiethnic breast cancer patients in this study appears to be substantially higher than previously reported [10, 15-17]. This may be explained by the high burden of risk factors of CVD in the background multiethnic population of Southeast Asia as have been reported in Malaysia, as well as Singapore [18-19]. The present observations warrant concern given that in the Life after Cancer Epidemiology Study, hypertension was independently associated with an increased risk of all-cause mortality among early-stage breast cancer survivors due to causes both related and unrelated to cancer [20]. A previous study had also shown that breast cancer patients with diabetes were more likely to be hospitalised for chemotherapy-induced toxicity than their counterparts without diabetes, leading to higher cancer specific mortality (hazard ratio (HR):1.20, 95\% Cl: 1.07-1.35) [21]. More recently, Hershman et al [22] through an analysis of data from clinical trials of women with breast cancer had shown that presence of every additional risk factor of CVD at baseline was associated with increased incidences of cardiac events and mortality.

The ethnic differences in prevalence of risk factors of CVD, and predicted risks of cardiotoxicity among women with breast cancer in the present study to some extent may explain the disparities in survival following breast cancer between the Malay, Indian and Chinese patients that we had previously reported from Southeast Asia [23]. This is further corroborated by findings that comorbidities including diabetes and hypertension, explained up to $50 \%$ of the survival disparities between the black and white women diagnosed with breast cancer in the United States [24]. Physicians should therefore be mindful that ethnicity might play a role as a prognostic factor in breast cancer through several mechanisms. 
Table 3. Association between baseline cardiovascular profile and adjuvant treatment administration in 1,876 women with non-metastatic breast cancer.

\begin{tabular}{|c|c|c|c|c|}
\hline \multirow{2}{*}{$\begin{array}{l}\text { Cardiovascular profile at initial } \\
\text { cancer diagnosis }\end{array}$} & \multicolumn{4}{|c|}{ Adjuvant therapy administration } \\
\hline & $\begin{array}{l}\text { OR for chemotherapy } \\
\qquad(95 \% \mathrm{Cl})^{\mathrm{a}}\end{array}$ & $\begin{array}{l}\text { OR for radiotherapy } \\
\qquad(95 \% \mathrm{Cl})^{\mathrm{b}}\end{array}$ & $\begin{array}{l}\text { OR for endocrine therapy } \\
\qquad(95 \% \mathrm{Cl})^{c}\end{array}$ & $\begin{array}{l}\text { OR for trastuzumab } \\
(95 \% \mathrm{Cl})^{\mathrm{d}}\end{array}$ \\
\hline \multicolumn{5}{|l|}{ Pre-existing CVDe } \\
\hline Yes & $0.32(0.17-0.58)$ & $0.63(0.37-1.08)$ & $0.47(0.21-1.09)$ & - \\
\hline No & 1.00 & 1.00 & 1.00 & - \\
\hline \multicolumn{5}{|l|}{$\begin{array}{l}\text { Presence of two or more } \\
\text { modifiable risk factors of } C V D^{f}\end{array}$} \\
\hline Yes & $0.95(0.71-1.28)$ & $1.12(0.86-1.44)$ & $1.60(1.10-2.50)$ & $0.67(0.39-1.15)$ \\
\hline No & 1.00 & 1.00 & 1.00 & 1.00 \\
\hline \multicolumn{5}{|l|}{$\begin{array}{l}\text { Family history of early-onset } \\
\text { coronary heart disease }^{\mathrm{g}}\end{array}$} \\
\hline Yes & $0.89(0.59-1.36)$ & $1.22(0.83-1.78)$ & $1.02(0.55-1.88)$ & $1.31(0.69-2.82)$ \\
\hline No & 1.00 & 1.00 & 1.00 & 1.00 \\
\hline
\end{tabular}

aThis analysis included 1,752 women of whom 1,105 received adjuvant chemotherapy (124 patients with unknown chemotherapy status were excluded). Results were derived using a multivariable logistic regression model including preexisting CVD, presence of $\geq 2$ risk factors of CVD and family history of early-onset coronary heart disease, adjusted for age (years), ethnicity (Chinese, Malay, Indian, others), type of hospital (public, private, university), tumour size category ( $\mathrm{pT} 1, \mathrm{pT}$ 2, pT3, pT4), lymph node status (pN0, pN1, pN2, pN3), oestrogen/progesterone receptor status (positive, negative), human epidermal growth factor receptor 2 status (positive, negative), tumour grade (I, II, III), adjuvant radiotherapy administration (no, yes), adjuvant endocrine therapy administration (no, yes) and trastuzumab administration (no, yes)

'This analysis included 1,749 women of whom 1,104 received adjuvant radiotherapy (127 patients with unknown radiotherapy status were excluded). Results were derived using a model similar as in (a) but mutually adjusted for adjuvant chemotherapy status, adjuvant endocrine therapy status and trastuzumab administration

'This analysis included 1,790 women of whom 1,115 received adjuvant endocrine therapy (86 patients with unknown endocrine therapy status were excluded). Results were derived using a model similar as in (a) but mutually adjusted for adjuvant chemotherapy status, adjuvant radiotherapy status and trastuzumab administration

'This analysis included 1,834 women of whom 126 received trastuzumab (42 patients with unknown trastuzumab administration status were excluded). Results were derived using a multivariable logistic regression model including presence of $\geq 2$ risk factors of CVD and family history of early-onset coronary heart disease, adjusted for age, type of hospital, human epidermal growth factor receptor 2 status, adjuvant chemotherapy status, adjuvant radiotherapy administration and adjuvant endocrine therapy status

${ }^{e}$ Comprises angina, myocardial infarction, stroke, atrial fibrillation, valvular diseases, heart failure, cardiomyopathy and rheumatic heart disease fIncludes hypertension, diabetes mellitus, obesity, dyslipidaemia and smoking

${ }^{\mathrm{g}}$ Defined as coronary heart disease diagnosed before the age of 60 years in a first-degree relative

In the current study, we have shown that presence of pre-existing CVD such as myocardial infarction and stroke appeared to be consistently associated with lower administrations of adjuvant therapies. Our observation is in keeping with results from a large population-based study in Canada, which also showed that women with breast cancer who had pre-existing myocardial infarction, congestive heart failure, arrhythmia or cerebrovascular accidents were less likely to receive the recommended treatment for their cancer including chemotherapy (OR: 0.56; $95 \%$ $\mathrm{Cl}: 0.48-0.66)$ and radiotherapy (OR: 0.75; 95\% Cl: 0.67-0.83) [25].

Our findings that clustering of modifiable cardiovascular risk factors such as hypertension, diabetes, obesity and dyslipidaemia was not associated with administration of most adjuvant therapies corroborate the notion that these risk factors are presently not factored into cancer treatment decision-making [9]. This however is not unexpected given the prevailing uncertainties on how information on conventional cardiovascular comorbidities including hypertension and diabetes may be useful in improving adjuvant treatment decision-making in oncology practices. The lack of association between cardiovascular risk and administration of cancer therapies does not allude to a need to change 
clinical practices in terms of prescribing adjuvant therapies. Instead, our findings support the pressing need to develop and facilitate stringent cardiac follow-up, surveillance of cardiac-related complications and collaborative multi-disciplinary care. To this end, cardiotoxicity prediction models may offer an avenue to incorporate data on patient-related cardiovascular factors with cancer treatment-related factors leading to estimation of cardiotoxicity scores to facilitate risk stratification of newly diagnosed cancer patients. Such an approach may potentially enable early identification of high-risk individuals whom may benefit from optimisation of anticancer therapies, early cardiology referral, close cardiovascular follow-up and participation in cardiac rehabilitation programmes (e.g. structured exercise therapy and nutritional counselling) [8].

However, there is presently an unmet need for accurate cardiovascular prognostic prediction rules for cancer patients [10]. For instance, while the staggering burden of cardiovascular risk factors at initial diagnosis coupled with administration of anthracyclines appears to put women with breast cancer in the present study at high a priori risks of developing cardiotoxicity, the Cardiotoxicity Risk Score that we had used to estimate the above is yet to undergo external validation [11]. It must also be noted that in order to develop accurate cardiotoxicity risk prediction models for use in oncology practices, high-quality longitudinal data that include detailed data on patients' cardiovascular profiles are needed apart from their cancer specific data. These will not only allow investigators to verify and quantify the prognostic impact of the individual cardiovascular risk factors on occurrence of cardiotoxicity, and overall and cancer-specific mortalities prior, but also enable the external validation of newly developed cardiotoxicity risk models.

The strengths of the current study include our prospective design where cardiovascular comorbidities and their influence on adjuvant treatment administration have been examined in an unselected cohort of newly diagnosed breast cancer patients, in which the biomarkers were measured at baseline prior to adjuvant treatment decision-making. Nonetheless, while we are in a position to follow-up patients over long term to measure the association between the individual cardiovascular risk factors and survival outcomes, we are unable to measure the incidence of cardiotoxicity per se, and other cardiac outcomes in this inception cohort study due to a lack of resources.

Table 4. Predicted risk of cardiotoxicity following adjuvant therapy in women with non-metastatic breast cancer.

\begin{tabular}{|c|c|c|c|c|c|c|c|c|c|}
\hline & \multicolumn{8}{|c|}{ Predicted risk of cardiotoxicity } & \multirow{4}{*}{ OR $(95 \% \mathrm{Cl})^{\mathrm{b}}$} \\
\hline & \multicolumn{2}{|c|}{ Low } & \multicolumn{2}{|c|}{ Intermediate } & \multicolumn{2}{|c|}{ High } & \multicolumn{2}{|c|}{ Very high } & \\
\hline & $n$ & $\%$ & $n$ & $\%$ & $n$ & $\%$ & $n$ & $\%$ & \\
\hline Overall & 340 & 19.9 & 282 & 16.5 & 577 & 33.7 & 511 & 29.9 & \\
\hline \multicolumn{10}{|c|}{ Age group } \\
\hline$<40$ & 29 & 15.8 & 9 & 4.9 & 114 & 62.3 & 31 & 16.9 & $6.61(4.05-10.79)$ \\
\hline $40-49$ & 113 & 24.3 & 32 & 6.9 & 214 & 46.0 & 106 & 22.8 & $3.97(2.79-5.66)$ \\
\hline $50-64$ & 171 & 22.8 & 103 & 13.7 & 202 & 26.9 & 274 & 36.5 & $2.40(01.77-3.26)$ \\
\hline$\geq 65$ & 027 & 08.7 & 138 & 44.2 & 047 & 15.1 & 100 & 32.1 & 1.00 \\
\hline \multicolumn{10}{|l|}{ Ethnicity } \\
\hline Malay & 076 & 11.7 & 085 & 13.1 & 249 & 38.4 & 238 & 36.7 & $01.29(0.94-1.76)$ \\
\hline Chinese & 236 & 29.1 & 148 & 18.2 & 248 & 30.6 & 179 & 22.1 & 1.00 \\
\hline Indian & 023 & 10.5 & 041 & 18.6 & 070 & 31.8 & 086 & 39.1 & $01.43(0.97-2.11)$ \\
\hline Others & 005 & 16.1 & 008 & 25.8 & 010 & 32.3 & 008 & 25.8 & $00.64(0.28-1.47)$ \\
\hline \multicolumn{10}{|c|}{ TNM cancer stage } \\
\hline I & 179 & 42.9 & 111 & 26.6 & 088 & 21.1 & 039 & 09.4 & 1.00 \\
\hline II & 115 & 15.2 & 118 & 15.6 & 284 & 37.5 & 240 & 31.7 & $04.82(03.65-6.36)$ \\
\hline III & 041 & 07.8 & 051 & 09.7 & 204 & 38.6 & 232 & 43.9 & $10.13(07.29-14.07)$ \\
\hline
\end{tabular}

${ }^{a}$ A priori risk of cardiotoxicity was estimated using the Cardiotoxicity Risk Score, which incorporates drug-related and patient-related risk factors [11]

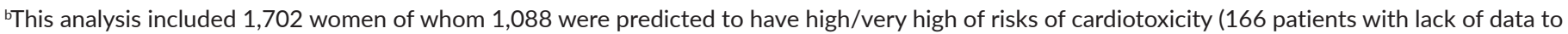
compute Cardiotoxicity Risk Score were excluded). Results were derived using logistic regression analysis with high/very high risk of cardiotoxicity as outcome of interest, and age, ethnicity, type of hospital and TNM cancer stage as covariates 


\section{Conclusion}

While many professional guidelines including those from the American Society of Clinical Oncology [12] and the European Society of Medical Oncology [26] have recommended routine cardiovascular evaluation of adult patients with cancer prior to initiation of potentially cardiotoxic therapies, the present findings allude to a gap in knowledge on whether data on burden of traditional cardiovascular risk factors may be useful in optimisation of cancer treatment decision-making. Although use of cardiotoxicity risk prediction tools may aid the above, more research is needed in the development and validation of accurate tools.

From a regional perspective, this study highlights the pressing need to accelerate the establishment of coordinated partnerships between the oncology and cardiology specialities to improve cardiovascular outcomes following breast cancer in the low- and middle-income settings. Although it is currently unclear whether aggressive management of modifiable risk factors of CVD may reduce the risk of cancer therapyinduced cardiotoxicity, it is conceivable that optimal control of these factors may lower the risk of cardiovascular events leading to improved overall survival following breast cancer [7, 21-22]. Notably, in resource-limited settings, amidst the shortage of oncologists and cardiologists, primary care physicians might potentially play an essential role in managing risk factors of CVD in cancer survivors.

\section{Conflicts of interest}

The authors have no conflict of interest to report.

\section{Funding}

The authors have no funding to declare for this work.

\section{References}

1. Zaorsky NG, Churilla TM, and Egleston BL, et al (2017) Causes of death among cancer patients Ann Oncol $28400-407$ https://doi. org/10.1093/annonc/mdw604

2. Khouri MG, Douglas PS, and Mackey JR, et al (2012) Cancer therapy-induced cardiac toxicity in early breast cancer: addressing the unresolved issues Circulation 126 2749-2763 https://doi.org/10.1161/CIRCULATIONAHA.112.100560 PMID: 23212997 PMCID: 3667651

3. Thavendiranathan P, Abdel-Qadir H, and Fischer HD, et al (2016) Breast cancer therapy-related cardiac dysfunction in adult women treated in routine clinical practice: a population-based cohort study J Clin Oncol 34 2239-2246 https://doi.org/10.1200/ JCO.2015.65.1505 PMID: 27091709

4. Blaes $\mathrm{AH}$, Thavendiranathan $\mathrm{P}$, and Moslehi J (2018) Cardiac toxicities in the era of precision medicine: underlying risk factors, targeted therapies, and cardiac biomarkers Am Soc Clinl Oncol Educ Book 38 764-774 https://doi.org/10.1200/EDBK_208509

5. Hooning MJ, Botma A, and Aleman BMP, et al (2007) Long-term risk of cardiovascular disease in 10-year survivors of breast cancer J Natl Cancer Inst 99 365-375 https://doi.org/10.1093/jnci/djk064 PMID: 17341728

6. Accordino MK, Neugut Al, and Hershman DL (2014) Cardiac effects of anticancer therapy in the elderly J Clin Oncol 32 (24) 2654 https:// doi.org/10.1200/JCO.2013.55.0459 PMID: 25071122 PMCID: 4876340

7. Jones LW, Haykowsky MJ, and Swartz JJ, et al (2007) Early breast cancer therapy and cardiovascular injury J Am Coll Cardiol 50 14351441 https://doi.org/10.1016/j.jacc.2007.06.037 PMID: 17919562 
8. Gilchrist SC, Barac A, and Ades PA, et al (2019) Cardio-oncology rehabilitation to manage cardiovascular outcomes in cancer patients and survivors: a scientific statement from the American Heart Association Circulation 139 e997-e1012 https://doi.org/10.1161/ CIR.0000000000000679 PMID: 30955352 PMCID: 7603804

9. Coviello JS (2018) Cardiovascular and cancer risk: the role of cardio-oncology J Adv Pract Oncol 9 160-176 PMID: 30588351 PMCID: 6303003

10. Clark RA, Marin TS, and Berry NM, et al (2017) Cardiotoxicity and cardiovascular disease risk assessment for patients receiving breast cancer treatment Cardio-Oncology 36 https://doi.org/10.1186/s40959-017-0025-7 PMID: 32154001 PMCID: 7048103

11. Herrmann J, Lerman A, and Sandhu NP, et al (2014) Evaluation and management of patients with heart disease and cancer: cardiooncology Mayo Clin Proc 89 1287-1306 https://doi.org/10.1016/j.mayocp.2014.05.013 PMID: 25192616 PMCID: 4258909

12. Armenian SH, Lacchetti C, and Barac A, et al (2017) Prevention and monitoring of cardiac dysfunction in survivors of adult cancers: American Society of Clinical Oncology clinical practice guideline J Clin Oncol 35 893-911 https://doi.org/10.1200/JCO.2016.70.5400

13. Mitchell J, and Lenihan DJ (2018) Management of cancer-therapy-induced LV dysfunction: can the guidelines help? [https://www.acc. org/latest-in cardiology/articles/2018/10/30/09/08/management-of-cancer-therapy-induced-Iv-dysfunction]

14. Ministry of Health Malaysia (2017) Clinical Practice Guidelines. Management of Dyslipidaemia 5th edn [https://www.moh.gov.my/moh/ resources/Penerbitan/CPG/CARDIOVASCULAR/4.pdf]

15. Liu D, Ma Z, and Yang J, et al (2019) Prevalence and prognosis significance of cardiovascular disease in cancer patients: a populationbased study Aging 11(18) 7948 https://doi.org/10.18632/aging.102301 PMID: 31562288 PMCID: 6781987

16. Alsaeed E, Albeeshi M, and Alsari M, et al (2017) Diabetes mellitus, hypertension, hyperlipidemia and obesity do not affect tumour expression of estrogen and progesterone receptors in Saudi breast cancer patients Kuwait Med J 1(49) 17-21 [https://applications. emro.who.int/imemrf/Kuwait_Med_J/Kuwait_Med_J_2017_49_1_17_21.pdf]

17. Nechuta S, Lu W, and Zheng, et al (2013) Comorbidities and breast cancer survival: a report from the Shanghai breast cancer survival study Breast Cancer Res Treat 139 227-235 https://doi.org/10.1007/s10549-013-2521-2 PMID: 23605082 PMCID: 3712758

18. Institute for Public Health (IPH) (2015) National Health and Morbidity Survey 2015 (NHMS 2015). Vol. II: Non-Communicable Diseases, Risk Factors \& Other Health Problems (Kuala Lumpur: Ministry of Health Malaysia) [http://iku.moh.gov.my/images/IKU/Document/REPORT/ nhmsreport2015vol2.pdf]

19. Khoo CM, and Tai ES (2014) Trends in the incidence and mortality of coronary heart disease in Asian Pacific Region J Atheroscler Thromb 21 S2-S8 https://doi.org/10.5551/jat.21_Sup.1-S2

20. Braithwaite D, Moore DH, and Satariano WA, et al (2012). Prognostic impact of comorbidity among long-term breast cancer survivors: results from the LACE study Cancer Epidemiol Biomarkers Prev 21 1115-1125 https://doi.org/10.1158/1055-9965.EPI-11-1228 PMID: 22573797 PMCID: 3470873

21. Srokowski TP, Fang S, and Hortobagyi GN, et al (2009) Impact of diabetes mellitus on complications and outcomes of adjuvant chemotherapy in older patients with breast cancer J Clin Oncol 27 2170-2176 https://doi.org/10.1200/JCO.2008.17.5935 PMID: 19307509 PMCID: 2674004

22. Hershman DL, Till C, and Shen S, et al (2018) Association of cardiovascular risk factors with cardiac events and survival outcomes among patients with breast cancer enrolled in SWOG clinical trials J Clin Oncol 36 2710-2717 https://doi.org/10.1200/JCO.2017.77.4414 PMID: 29584550 PMCID: 6127026

23. Bhoo-Pathy N, Hartman M, and Yip CH, et al (2012). Ethnic differences in survival after breast cancer in South East Asia PLoS One 7 e30995 https://doi.org/10.1371/journal.pone.0030995 PMID: 22363531 PMCID: 3283591 
24. Tammemagi CM, Nerenz D, and Neslund-Dudas C, et al (2005) Comorbidity and survival disparities among black and white patients with breast cancer J Am Med Assoc 294 1765-1772 https://doi.org/10.1001/jama.294.14.1765

25. Abdel-Rahman O, Xu Y, and Kong S (2019) Impact of baseline cardiovascular comorbidity on outcomes in women with breast Cancer: a real-world, population-based study Clin Breast Cancer 19(2) e297-e305 https://doi.org/10.1016/j.clbc.2018.12.005 PMID: 30630679

26. Curigliano G, Lenihan D, and Fradley M (2020) Management of cardiac disease in cancer patients throughout oncological treatment: ESMO consensus recommendations Ann Oncol 31(2) 171-190 https://doi.org/10.1016/j.annonc.2019.10.023 PMID: 31959335 PMCID: 8019325 